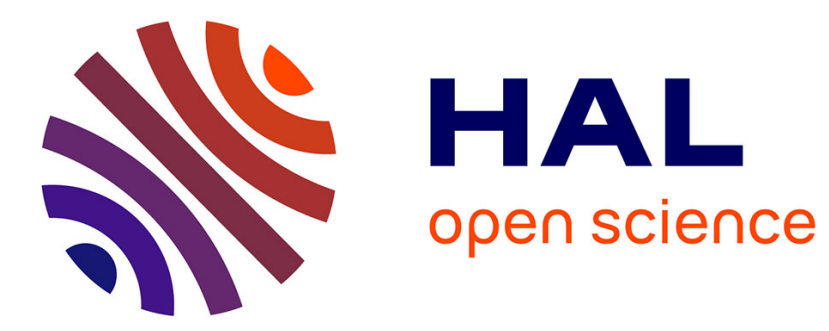

\title{
La Faculté des sciences de Lille pendant l'occupation allemande (1914-1918)
}

Patrick Auguste, Braun Sophie, Pourprix Marie-Thérèse

\section{To cite this version:}

Patrick Auguste, Braun Sophie, Pourprix Marie-Thérèse. La Faculté des sciences de Lille pendant l'occupation allemande (1914-1918). La Guerre des cartables 1914-1918, 2018. hal-02341380

\section{HAL Id: hal-02341380 \\ https://hal.science/hal-02341380}

Submitted on 6 Nov 2019

HAL is a multi-disciplinary open access archive for the deposit and dissemination of scientific research documents, whether they are published or not. The documents may come from teaching and research institutions in France or abroad, or from public or private research centers.
L'archive ouverte pluridisciplinaire HAL, est destinée au dépôt et à la diffusion de documents scientifiques de niveau recherche, publiés ou non, émanant des établissements d'enseignement et de recherche français ou étrangers, des laboratoires publics ou privés. 


\section{ท:

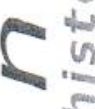 \\ 0

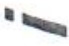 \\ $\downarrow$ \\ O) \\ n \\ -

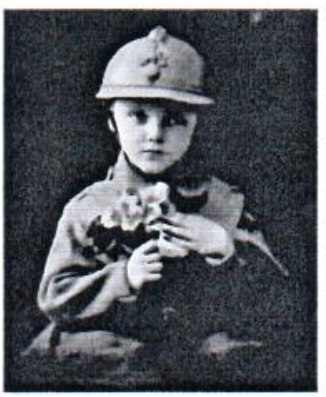 \\ Détail d'une carte postale intitulée a Mes \\ La Guerre des cartables \\ 1914-1918 \\ Élèves, étudiants et enseignants dans la Grande Guerre en Nord-Pas-de-Calais}

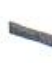

י

$>$

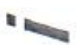

$U$

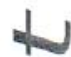

(1)

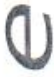

$\checkmark$

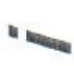

0

w

y

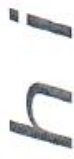

Les départements du Nord et du Pas-de-Calais, entre 1914 et 1918, vivent une situation très difficile. Environ $70 \%$ du département du Nord et $25 \%$ de celui du Pas-de-Calais connaissent une terrible occupation allemande. À l'ouest de la ligne de front, le territoire devient une zone stratégique pour les armées alliées, débarquant par Calais, Boulogne-sur-Mer et Dunkerque. Comment dans de telles conditions, marquées par la densité de la présence militaire et par la proximité du front, une activité scolaire et universitaire peut-elle se maintenir et pour quelles finalités ? Organisé autour de dix-neuf contributions et d'une présentation introductive, cet ouvrage décrit la manière dont les acteurs et les structures éducatives réussissent, au milieu des pires difficultés, à maintenir leurs activités, à la fois dans la zone occupée et dans la zone « alliée », luttant pour leur survie. Lorsque sonne l'heure de l'armistice, la lourdeur du bilan est obsédante pour l'École septentrionale qu'il faut reconstituer.
Jean-François Condette, professeur en histoire contemporaine, est membre du Laboratoire CREHS (EA 4027) de 'Université d'Artois et directeuradjoint de l'ESPE-Lille-Nord-deFrance (COMUE-LNF), en charge de la Recherche. II travaille sur 'histoire des structures et des acteurs éducatifs. Ses recherches portent également sur les élites de l'État et sur les occupations militaires dans la France septentrionale au XXe siècle.

\section{Contributeurs}

Patrick Auguste

Aldo Battaglia

François-Xavier Boone

Sophie Braun

Philippe Cadet

Jean-François Condette

Christine Dalbert

François Da Rocha Carneiro

Julien Dochez

Magali Domain

Delphine Dufour

Johann-Günther Egginger

Jean-Baptiste Gardon

Sandrine Gorez-Brienne

Jean-François Grevet

Jean Heuclin

Stéphane Lembré

Philippe Marchand

Marie-Thérèse Pourprix

Philippe Roger

Laurent Sellier

Christine Simonnet-Focquenoy

Corinne Vezirian-Lefeuvre

Isabelle Westeel

ISBN: 978-2-7574-2071-3 ISSN: $1284-565534$ € Maquetile tico as Delarginiliere

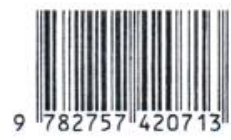




\section{La faculté des sciences de Lille pendant l'occupation allemande (1914-1918)}

Patrick Auguste, Sophie Braun et Marie-Thérèse Pourprix

Durant le premier conflit mondial, l'Université de Lille a la particularité, par apport à ses consœurs françaises, d'être la seule occupée par les troupes allemandes zu ce durant les quatre années de guerre, d'octobre 1914 à octobre 1918. La vie de - faculté des sciences n'en demeure pas moins tangible, malgré les conditions Aexercice difficiles liées à l'occupation de la Ville. Comment les membres de la inculté, le rectorat et la municipalité ont maintenu la mission d'enseignement z Ze recherche malgré les dégâts matériels importants, l'absence d'enseignants and ceudiants, l'impossibilité de se déplacer, de communiquer avec l'extérieur ? Qeel rôle a joué « l'étroite solidarité de tous les membres de la grande famille anversitaire ${ }^{1}$ » pour le maintien continuel des cours, des examens, du travail scientifique, des collections? Quelles connaissances les scientifiques lillois ont zo apporter et développer au cours de la guerre, au service de la recherche ?

\section{Lic contexte de l'occupation et de la guerre stson impact sur la faculté}

Lersque la guerre éclate en 1914, l'Université de Lille réunit la faculté des siences, la faculté de droit, la faculté des lettres, la faculté de médecine et de narmacie ainsi que la bibliothèque universitaire et la maison des étudiants. La - Taiorité de ses bâtiments sont regroupés depuis la fin du XIX ${ }^{\mathrm{e}}$ siècle au sein An quartier latin lillois dans le nouveau quartier Saint-Michel, avec son siège aministratif situé Place Philippe Le Bon.

Tal CHARMEIL, « Rapport sur la situation de l'enseignement supérieur à Lille pendant 7année scolaire 1914-I915 》, dans Annales de l'Université de Lille : rapport annuel du Conseil LI'Université..., Lille, Imprimerie Le Bigot, p. Io. 


\section{La faculté des sciences de Lille face à l'entrée en guerre}

Dans ce quartier, la faculté des sciences englobe les instituts de mathématiques, de physique, de sciences naturelles - comprenant les services de zoologie, géologie et botanique - et de chimie. Plus loin, dans les anciens locaux de la faculté, rue des Fleurs, l'Institut électrotechnique trouve place. Des relations privilégiées existent avec l'Institut industriel du Nord et l'École nationale d'Arts et Métiers de Lille. Enfin, l'Observatoire de Lille situé à Hem et le laboratoire maritime du Portel y sont rattachés.

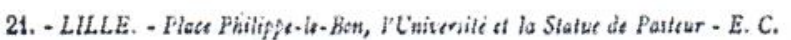

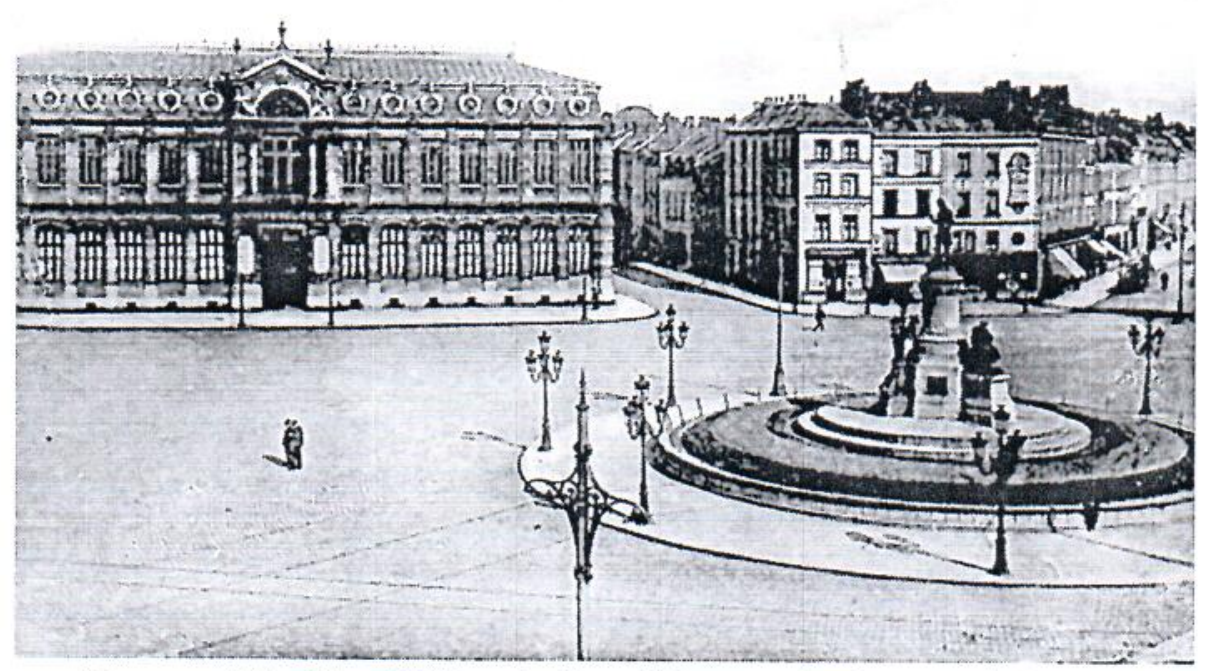

Figure 7 : Lille, Place Philippe Le Bon, l'Université et la statue de Pasteur, carte postale d'époque, fin XIX $\mathrm{X}^{\mathrm{e}}$ - début $\mathrm{XX}^{\mathrm{e}}$ siècle. Collection particulière.

Sans revenir en détail sur les débuts de celle qui sera nommée « la Grande Guerre », quelques dates sont à mentionner. Le $\mathrm{I}^{\mathrm{er}}$ août, la France appelle à la mobilisation après la déclaration de guerre de l'Allemagne à la Russie. Le même jour, Lille est déclarée « ville ouverte » : le ministre de la Guerre ordonne de ne pas défendre la ville. À cette époque, Lille est encore entourée par l'enceinte construite par Vauban, avec des entrées/portes uniquement en certains points. Le 2, le Luxembourg est envahi par l'Allemagne, qui pose par ailleurs un ultimatum à la Belgique. Le 3, l'Allemagne déclare la guerre à la Belgique et à la France. Le 4, elle envahit la Belgique, ce qui déclenche l'entrée du Royaume-Uni en guerre, contre l'Allemagne. Le 5, la bataille de Liège débute. Les événements s'enchaînent et une véritable « bataille des frontières » s'opère durant lc mois d'août. Le 25, la ville de Louvain est incendiée : la bibliothèque et les archives de l'Université, une des plus anciennes d'Europe, sont ravagées. Le retentissement de cet acte choquant, qualifié de «barbare », est mondial et le courage des Belges qui résistent est salué.
Le z septembre.

Eass combat. Le s, L

aciei bommes mobil

vaumes. Du 6 au is

Ais est a nouveau o

Eactobre. Lille est er

nume ecrite circule, er

ars atciormés de fuir

lencemi. Un véritab

ax sosenvirons, afind

ume grande partie du

rtwoules vers Lille pa

aure de l'Observato

Ae la zone occupee.

vous pour ce trajet d

teutes $\%$, marchant $d$

trecru a Boulogne as:

wile de Lille est bom

ap aposee dans la V

ziss a la population ».

ano (méme si la régil

and pas ces écrits ras

12 Ville et ses habitar

L'impact de la gt

les dégâts matéri

Des le 3 octobre 19

L'Observatoire de l'1

oxmme en témoigner

coupole du bâtiment

nambe sur l'Institut i.

shrapnel et contenant

sit de l'Institut des si

de l'Institut géologio

er le cabinet du prép

Des dégâts sont aussi

pluie. Le 28 novembr

=- - $b: d$, p. 6.

- Jean-Claude THORE:

present, 2009 , p. $162 \cdot 1$

- Ibid., p. I66.

- Benoît Côme DAMIE:

Faculté pendant l'anne

annuel du Conseil de I"t

6. - Charles BARROIS,

la Société Géologique 2 is 
Le 2 septembre, un détachement de l'armée allemande pénètre dans Lille

ues, de ologie té, rue légiées Métiers me du

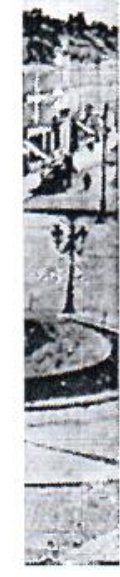

$1 \mathrm{r}$,

̀̀re.

irande lle à la même une de - ceinte oints. irs un = et à la ıe-Uni ments ? mois ves de ement ge des sans combat. Le s, Lille est occupée par les Allemands qui offrent la possibilité aux hommes mobilisables de quitter la ville. L'occupation ne dure qu'une journée. Du 6 au is septembre, la contre-offensive de la Marne a lieu. Le 12, Lille est à nouveau occupée, toujours sans installation solide des Allemands. Le 2 octobre, Lille est encore investie par les Allemands. À partir du 9, une consigne non écrite circule, enjoignant les classes mobilisables non encore mobilisées ou les réformés de fuir la zone occupée pour éviter de se retrouver à la merci de l'ennemi. Un véritable exode des hommes mobilisables a lieu, à partir de Lille et de ses environs, afin de gagner la zone non occupée. La consigne est appliquée par une grande partie du personnel et des étudiants de l'Universitéz. Certains sont refoulés vers Lille par les Allemands. Le récit de Robert Jonckheere, propriétaire de l'Observatoire de Lille, témoigne des difficultés rencontrées à sortir de la zone occupée. Il raconte son périple jusqu'à Londres : «j'avais mis cinq jours pour ce trajet de Lille à Londres, qu'en temps ordinaire, je faisais en cinq heures », marchant des kilomètres, empruntant des trains bondés, puis enfin un bateau à Boulogne après avoir tenté un départ à Calais ${ }^{3}$. Du 10 au 13 octobre, la ville de Lille est bombardée. Le I2, la chute de Lille est annoncée et une affiche est apposée dans la Ville : « l'armée allemande ne fait la guerre qu'aux armées, pas à la population ». Le souvenir de telles promesses déjà faites et non tenues en I870 (même si la région lilloise a été épargnée par l'occupation en 1870-1871) ne rend pas ces écrits rassurants. À partir du I3, Lille est aux mains des Allemands. La Ville et ses habitants subiront le régime d'Occupation jusqu'en octobre 1918.

\section{L'impact de la guerre sur les locaux de la faculté : les dégâts matériels}

Dès le 3 octobre 1914, la faculté des sciences est touchée par la guerre. L'Observatoire de l'Université de Lille à Hem est occupé par les Allemands, comme en témoignent deux photographies figeant les soldats allemands sur la coupole du bâtiment ${ }^{4}$. Lors des bombardements du to au 13 octobre, un obus tombe sur l'Institut industriel' et un obus à balles (plus connu sous le nom de shrapnel et contenant de nombreuses billes de métal servant de balles) perce le toit de l'Institut des sciences naturelles. Ce dernier éclate dans l'étage supérieur de l'Institut géologique et démolit la salle des dons, des sondages, le magasin et le cabinet du préparateur et répand ses projectiles dans tout le bâtiment ${ }^{6}$. Des dégâts sont aussi causés par l'effondrement de cheminées, de cloisons et la pluie. Le 28 novembre, un incendie ravage l'Institut électrotechnique, rendant

2.- Ibid., p. 6.

3.- Jean-Claude THOREL, Le ciel d'une Vie. Robert Jonckheere, Agnières, Éditions Le temps présent, 2009 , p. I62-I 68 .

4.- Ibid., p. 166.

5.- Benoît Côme DAMIEN, « Faculté des sciences : Rapport sur la situation et les travaux de la Faculté pendant l'année scolaire 1914-1915 », dans Annales de l'Université de Lille : rapport annuel du Conseil de l'Université..., Lille, Imprimerie Le Bigot, p. 33-34.

6.- Charles BARROIS, « La Société Géologique du Nord pendant la guerre », dans Annales de la Société Géologique du Nord, tome XLIV, r919, p. XII. 
impossible son utilisation'. Les cours sont transférés à l'Institut de physique En mars 1915, la Maison des étudiants, rue de Valmy, est réquisitionnée pour y organiser des conférences pour les officiers allemands ${ }^{8}$. En octobre, l'Institur de physique est mis à disposition d'un officier allemand, qui a le droit d'utiliset les laboratoires et appareils pour ses expériences9. Le II janvier 1916, le dépôt de munitions des « I8 Ponts 》 explose à proximité des bâtiments universitaires. Les bâtiments des sciences naturelles sont de nouveau gravement touchés: les vitres éclatent, les fenêtres et les portes tombent, les instruments de recherche et les collections sont gravement endommagés ${ }^{10}$. Les salles du musée Gosselet et du musée houiller ont particulièrement souffert : « les beaux plans du bassin houiller du Nord en perspective sur verre à l'échelle $\mathrm{I} / 10000^{\mathrm{e}}$ sont en pièces $^{11}$ ». Les services municipaux de Lille et le monde enseignant unissenc leurs efforts pour colmater les dégâts, faire reprendre les enseignements des instituts et protéger les biens ${ }^{12}$. La disparition d'Ernest Verbièse, le garçon du musée tué à l'ennemi en I9Is rend le sauvetage des collections difficile et le doyen honoraire Jules Gosselet entreprend l'opération. Le 20 mars, il décède, affaibli par l'émotion, la fatigue et le froid qui ont accompagné la remise en état des collections de son musée de géologie, dispersées par la catastrophe.

Le 25 avril 1916, un terrible incendie ravage l'Hôtel de Ville de Lille et la bibliothèque municipale. Les « débris », 80000 volumes, sont transférés à la bibliothèque universitaire, rue Jean Bart ${ }^{13}$, qui sert de refuge à de nombreuses collections, comme la bibliothèque du mathématicien Gustave Demartres expulsé de sa demeure. Le prêt des livres fonctionne de façon ininterrompue pendant toute la guerre grâce à la présence du bibliothécaire en chef. Le I 4 août 1917, l'Hôtel académique, où le recteur Georges Lyon et son épouse résident, est bombardé. Le couple en sort sain et sauf ${ }^{14}$.

7.-Ibid., p.3I.

8.- Jean-François CONDETTE, « L'Université de Lille dans la Première Guerre mondiale 1914-1918 》, Guerres Mondiales et Conflits Contemporains, mars 2000, $\mathrm{n}^{\circ}$ 197, Images civiles de la France en Guerre, p. 87.

9.- Alphonse MALAQUIN, « Rapport sur la situation de l'enseignement supérieur à Lille pendant l'année scolaire 1915-1916 », dans Annales de l'Université de Lille : rapport annuel du Conseil de l'Université..., Lille, Imprimerie Le Bigot, p. 46.

Io.- Ibid., p. 48.

II.- Benoît Côme DAMIEN, « Faculté des sciences : Rapport sur la situation et les travaux de la Faculté pendant l'année scolaire 1915-1916 », dans Annales de l'Université de Lille : rapport annuel du Conseil de l'Université..., Lille, Imprimerie Le Bigot, p. 93.

12.- Ibid., p. 89-94.

13.- Alphonse MALAQUIN, « Rapport sur la situation de l'enseignement supérieur à Lille pendant l'année scolaire 1915-1916 », dans Annales de l'Université de Lille: rapport annuel du Conseil de l'Université..., Lille, Impr. Le Bigot, p. 49-50.

I4.- Jules DEROCQUIGNY, « Rapport sur la situation de l'enseignement supérieur à Lille pendant l'année scolaire 1916-1917 », dans Annales de l'Université de Lille: rapport annuel du Conseil de l'Université..., Lille, Impr. Le Bigot, p. 102.

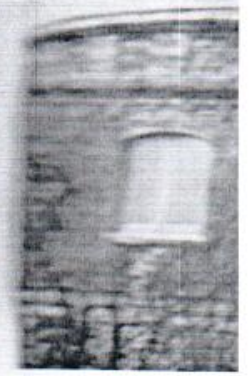

Fig

Les réorga

A partir de la

mois ordres d';

Les Allemand:

et des locaux u

la solidarité fo

hebergées à la

les instituts de jeunes filles sor de l'Institut de du Conseil de seurs de la fact caculté, et Alpl

Les réuni conviés de durant l'a trouvé l'o des nouve joie, parve

15.- Alphonse M.: pendant l'ans du Conseil de. :6.-Ibid., p. 46 es 17.-Ibid., p. 46 


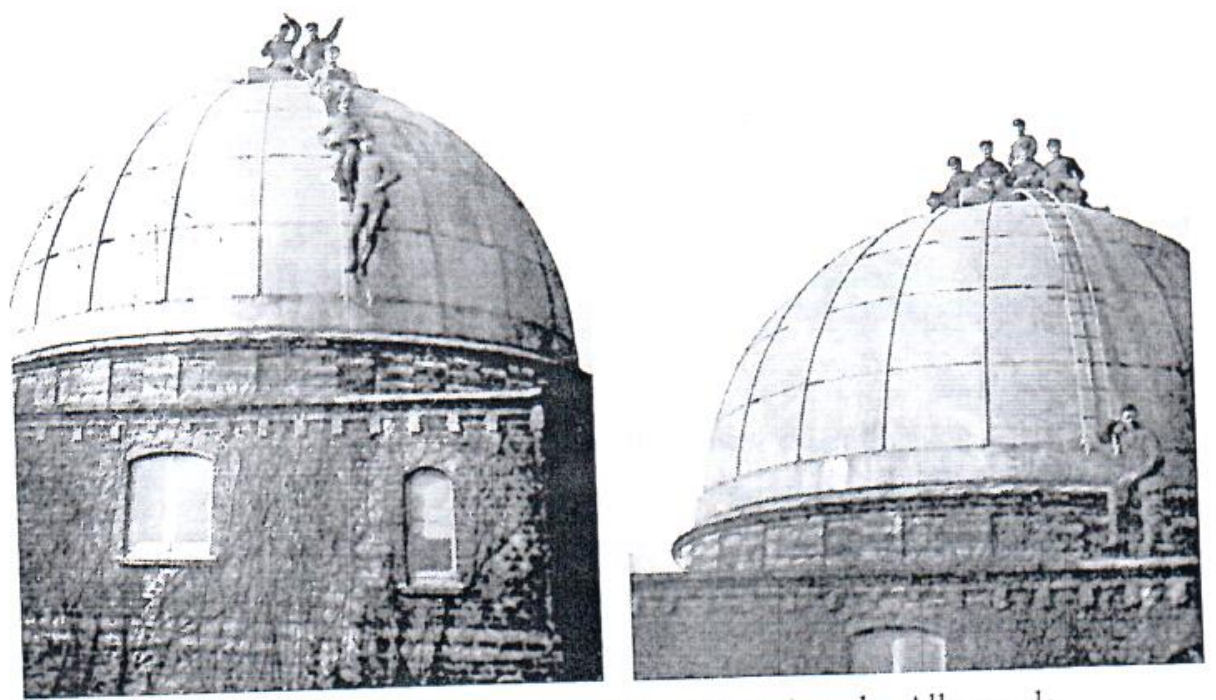

Figure 8: L'Observatoire de Hem occupé par les Allemands.

(c) Collection Y. Georgelin, dans Jean-Claude Thorel, Le ciel d'une Vie. Robert Jonckbeere, 2009 , p. 166.

\section{Les réorganisations en fonction des réquisitions et incendies}

À partir de la rentrée de novembre 1915, l'Université assure la liaison entre les trois ordres d'enseignement de la région : primaire, secondaire, universitaire ${ }^{15}$. Les Allemands occupant de nombreuses institutions, dont le lycée Faidherbe et des locaux universitaires, les liens institutionnels et fraternels se resserrent et la solidarité fonctionne de façon tangible. Les classes du lycée Faidherbe sont hébergées à la faculté des lettres avec les cours de physique et de chimie dans les instituts de la faculté des sciences. Celles de l'École primaire supérieure de jeunes filles sont accueillies à l'Institut de mathématiques, tout comme les élèves de l'Institut des jeunes aveugles et sourds-muets de Ronchin ${ }^{16}$. Enfin, les séances du Conseil de l'Université se tiennent à l'Hôtel de l'Académie ${ }^{17}$. Deux professeurs de la faculté des sciences y siègent : Benoît-Côme Damien, le doyen de la faculté, et Alphonse Malaquin.

Les réunions universitaires, instituées par M. le Recteur, et auxquelles sont conviés des représentants des trois ordres de l'enseignement, se sont poursuivies durant l'année scolaire 1915-1916. Les assistants, toujours nombreux, y ont trouvé l'occasion de suivre l'activité universitaire à tous les degrés. Parfois, des nouvelles rares et brèves, mais accueillies avec un grand sentiment de joie, parvenaient d'établissements éloignés du siège académique et venaient

I5.- Alphonse MALAQUIN, « Rapport sur la situation de l'enseignement supérieur à Lille pendant l'année scolaire I9I5-I916 », dans Annales de l'Université de Lille: rapport annuel du Conseil de l'Université..., Lille, Impr. Le Bigot, p. 45.

I6.- Ibid., p. 46 et p. 80.

17.-Ibid., p. 46 . 
attester que la mission éducatrice continue à s'exercer même dans les coins les plus reculés de nos régions. Toujours on y suivait avec attention les efforts courageux et ininterrompus de ceux qui ont la tâche, souvent rendue difficile, d'assurer le maintien de la vie scolaire. La multiplicité des problèmes qui se présentent dans la vaste organisation de l'enseignement primaire, les luttes qu'on est obligé d'y soutenir contre les événements quotidiens et imprévus, de même que les efforts de l'enseignement secondaire, étaient suivis avec un intérêt attentif par les membres de la réunion. Tous y sentaient mieux l'utilité d'une action solidaire et chacun y puisait un stimulant pour continuer sa tâche avec plus d'ardeur et plus de confiance. Lille, décembre $1916^{18}$.

En février 1917, les autorités allemandes ordonnent la fermeture des facultés en raison de la pénurie de charbon. Cette ordonnance ne sera pas appliquée grâce aux mesures prises par le recteur Georges Lyon, qui met ses appartements particuliers à la disposition des facultés, du 26 février au 16 avril. En juin 1917 , les cours sont interrompus de façon anticipée par une mesure de l'occupant qui envoie des hommes de quatorze à soixante ans subir un examen médical pour aller travailler dans une autre région. Les sessions d'examen et de baccalauréat sont organisées d'urgence. Le résultat est heureux : les étudiants passent leurs examens et leur évacuation forcée est finalement annulée. En octobre 1917 et mars 1918, tout laissez-passer est refusé entre Roubaix, Tourcoing et Lille, ce qui empêche la circulation de certains enseignants vers les facultés et les résignent à organiser des cours chez eux ${ }^{19}$. Aux mois d'avril et mai 1918, la réquisition des locaux de la faculté des lettres entraîne le transfert des cours dans des petites salles de la faculté des sciences : les services de zoologie et de botanique migrent vers les salles de travaux pratiques, tandis que les collections précieuses, herbiers et microscopes se retrouvent entassés dans les caves. En juin, Pierre Pruvost, bibliothécaire de la Société géologique du Nord installée dans les locaux de l'Institut de géologie, mentionne l'affiche réglementant l'accès à la bibliothèque de l'Institut de géologie : « 3 juin 1918 [...] L'avis explicatif suivant est placé sur la salle des livres et cartes : L'Institut géologique est pris par la Kommandantur. Il est défendu d'emporter des livres ou des cartes, mais c'est permis d'employer la bibliothèque. $[. ..] \gg^{20}$. La ville de Lille est devenue pour les Allemands un centre de services géologiques, hydrologiques et topographiques (service de la Kriegsgeologie) important et la mise à disposition de l'Institut de géologie (des cartes en particulier) s'avère importante. La même année, l'Institut de physique sert de caserne aux soldats du service des chemins de fer. De même, l'Institut de mathématiques est pillé, mis pratiquement hors d'usage. Des salles y sont transformées en dortoirs, cantine et lieu de détente pour les téléphonistes allemands ${ }^{21}$.

18.- Ibid., p. 63.

I9.- Jules JACQUEY, « Rapport sur la situation de l'enseignement supérieur à Lille pendant l'année scolaire 1917-1918 », dans Annales de l'Université de Lille: rapport annuel du Conseil de l'Université..., Lille, Imprimerie Le Bigot, p. I44.

20.- Collection de la Bibliothèque de l'UMR 8198 CNRS - Université de Lille Sciences et Technologies, Société géologique du Nord, Carnet de la bibliothèque.

2I.- Jules JACQUEY, « Rapport sur la situation de l'enseignement supérieur à Lille pendant l'année scolaire 1917-1918 », dans Annales de l'Université de Lille : rapport annuel du Conseil de l'Université..., Lille, Imprimerie Le Bigot, p. I45.

\section{Le main pourlat}

En dépit missiond I9I4 enjoi leurs tâch،

La réo

Bien que $\mathrm{F}$ lieu qu'en cultés d'or été enclin trouvé d'él s'établit et d'avec le rs tence impr début du n de la situai Allemands surtout au L'improvis rement. Le palement $\mathrm{r}$ manques $d c$ Devant les: d'examens manquants au cours des sements sor assurent les et de garçor la culture fr ciper à l'effc discours de l'Universite

Notre $\mathrm{C}$ elle dos autant

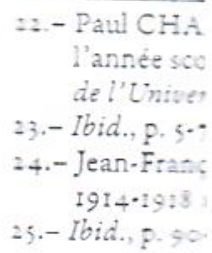




\section{Le maintien de la mission d'enseignement pour la faculté des sciences de Lille}

En dépit de ces événements, les universitaires présents à Lille poursuivent leur mission d'enseignement et de travail scientifique. Une circulaire du iz septembre I9I4 enjoint d'ailleurs les personnels de l'université et les étudiants à reprendre leurs tâches et leurs cours ${ }^{22}$.

\section{La réorganisation des cours}

Bien que programmée le 3 novembre, la rentrée de la faculté des sciences n'aura lieu qu'en janvier 19Is étant donné le chaos des débuts de la guerre et les difficultés d'organisation : «Dans ces conditions, à supposer que les maîtres eussent été enclins à reprendre leur enseignement, ils n'auraient certainement pas trouvé d'élèves pour les entendre. Ce n'est que peu à peu qu'une accoutumance s'établit et que les esprits réfléchis se firent à l'idée d'une longue séparation d'avec le reste du pays et à la nécessité d'une adaptation aux conditions d'existence imposées. Mais cela avait duré longtemps et l'on se trouvait arrivé au début du mois de février $1915^{23} \gg$. Les inscriptions ne sont pas prises en raison de la situation désordonnée et pour ne pas fournir des listes d'étudiants aux Allemands en recherche de main-d'œuvre ${ }^{2+}$. L'assiduité s'en ressent et il n'y aura, surtout au début de la guerre, que peu d'examens en dehors du baccalauréat. L'improvisation est permanente. Les lieux d'enseignements changent régulièrement. Les emplois du temps sont variables et légers. Les cours sont principalement regroupés en fin de matinée et en début d'après-midi en raison des manques de chauffage et d'éclairage et pour ne pas multiplier les déplacements. Devant les menaces allemandes de déportation de jeunes travailleurs, les sessions d'examens et de baccalauréat sont multipliées. Pour suppléer les personnels manquants (mobilisés, déportés), de multiples recrutements s'effectuent tout au cours des années de guerre. Des étudiants et des enseignants d'autres établissements sont embauchés, en particulier des professeurs agrégés de lycée. Ils assurent les fonctions de chargés de cours, de chefs de travaux, de préparateurs et de garçons de laboratoire. Par-dessus tout, la volonté de maintenir la vie et la culture française dans les facultés prime, s'agissant d'une manière de participer à l'effort de guerre français et de faire preuve de patriotisme ${ }^{25}$. Dans son discours de rentrée d'octobre 1915, Alphonse Malaquin montre la volonté de l'Université de remplir sa mission :

Notre Université n'a subi aucun contrôle et à l'heure où ces lignes sont écrites, elle doit affirmer qu'elle ne pourrait souffrir aucune ingérence étrangère autant dans le cours de son existence morale et intellectuelle que vis-à-vis de

22.- Paul CHARMEIL, « Rapport sur la situation de l'enseignement supérieur à Lille pendant l'année scolaire 1914-1915 », dans Annales de l'Université de Lille: rapport annuel du Conseil de l'Université..., Lille, Imprimerie Le Bigot, p. 5.

23.- Ibid., p. 5-7.

24.- Jean-François CONDETTE, « L'Université de Lille dans la Première Guerre mondiale 1914-191 $8 \gg$, Guerres Mondiales et Conflits Contemporains, mars 2000, n 197, p. 90.

25.-Ibid., p. 90-9r. 
son indépendance de grand établissement scientifique. Elle a pu être touchée matériellement par une mainmise impérieuse sur quelques-uns de ses bâtiments, mais elle se doit de vous dire que sa conscience lui aurait interdit de continuer son fonctionnement si le contrôle de l'autorité allemande s'était exercé [...]. Pendant sa longue séparation d'avec le pays, l'Université de Lille a suivi sa voie avec rectitude, guidée dans l'accomplissementde ses actes les plus simples comme les plus élevés, par le souci constant d'un patriotisme fidèle et vigilant ${ }^{26}$.

Ce point de vue est partagé par Charles Barrois, professeur de géologie, qui écrit la veille de l'armistice le Io novembre 1918 : « Pendant la guerre je me suis dévoué à mes étudiants - surtout à des étudiantes - qui désiraient prendre dans nos collèges la place des hommes disparus, et au sauvetage des collections ${ }^{27}{ }^{~}$.

À l'époque, l'enseignement se décline en certificats d'études supérieures (CES) préparatoires, préalables aux certificats de licence mathématiques générales (Math Géné) et sciences portant sur la physique, la chimie et l'histoire naturelle (SPCN, PCN), et en CES plus spécialisés. Le PCN fait office d'année propédeutique aux études médicales. Avec le diplôme d'ingénieur chimiste, ces certificats concentrent, pendant le conflit, la majorité des étudiants. Les certificats de licence (calcul différentiel et intégral, physique générale, physique industrielle, chimie générale, botanique, zoologie, géologie) sont maintenus et encadrés malgré un auditoire limité en nombre. Les CES plus spécialisés (analyse supérieure, géométrie supérieure, astronomie et mécanique céleste, géographie physique) et les diplômes d'études supérieures (DES) requis pour préparer l'agrégation ne sont que rarement enseignés. Les concours de l'agrégation et du certificat d'aptitude à l'enseignement secondaire ne sont pratiquement plus préparés. La priorité est donnée aux enseignements de base destinés aux plus jeunes, les plus âgés étant pour la plupart mobilisés. Le diplôme d'ingénieur chimiste continue d'être délivré. Le diplôme d'ingénieur électricien disparaît après l'incendie de 1914 et la plupart des étudiants susceptibles de s'inscrire pour le suivre sont mobilisés. Ce diplôme avait été mis en place vingt ans plus tôt pour former des « auxiliaires éclairés de la grande industrie électrique de construction et d'exploitation ${ }^{28}$.

Une des questions qui se pose concernant le lien entre la vie universitaire et économique dans Lille occupée est de savoir comment les enseignants, fonctionnaires, ont été confrontés à la coupure avec l'administration nationale, en particulier sur l'aspect des salaires. La diminution drastique de la monnaie courante

26.- Alphonse MALAQUIN, « Rapport sur la situation de l'enseignement supérieur à Lille pendant l'année scolaire 1915-1916 》, dans Annales de l'Université de Lille : rapport annuel $d u$ Conseil de l'Université..., Lille, Imprimerie Le Bigot, p. 43-44.

27.- WMD, dossier 31 (« Barrois, Charles Eugène »), lettre du ro novembre 1918, cité dans Nicolas GINSBURGER, « "La guerre, la plus terrible des érosions". Cultures de guerre et géographes universitaires Allemagne-France-États-Unis (1914-1921) ». Thèse Doctorat Université Paris Ouest Nanterre-La Défense, 2010, p. 75I.

28.- René SWYNGEDAUW, « L'Institut électrotechnique de l'Université de Lille », dans COLLECTIF, Lille et la région du Nord en Igog, Tome 1 : Lille : histoire, établissements d'instruction publique, musées, sociétés savantes, monuments, bygiène, commerce et industrie, Lille, Imprimerie Louis Danel, p. Io8.

et des liqu

de monna

municipal

a-dire ava

bons régic

Le cor

D'après le

rences ass:

Charles I

Buisine, E

Malaquin,

vingt et ut

Clairin, $R$

Paul Pasca

et cing gar

En phy

préparent

et une asf

agrégé de

fonctions

subsiste gr

mobilises.

soit des pe

pas le pass

René Swyn

appareils d

d'un labor

Parlement

considerab

En mat

par sept et:

rentiel et i:

rence comp

classe de m

des science

zrande imp

29,- Béatrice

occupé

la régula

30-- Aphons

pendant

du Conse

34--Benoit C

Faculte p

annuel du 
et des liquidités entraîne l'apparition de « monnaies de nécessité » sous forme de monnaies ou bons papiers remplaçant les francs. Ces bons sont émis par les municipalités pour le traitement des fonctionnaires dès le 3 I août 1914, c'esta-dire avant l'occupation de la ville ${ }^{29}$. Après la guerre, de février à mai 1919, ces bons régionaux seront remboursés en francs.

\section{Le corps professoral et l'enseignement}

D'après les Annales de l'Université, seuls douze professeurs et maîtres de conférences assurent le service en sciences à la rentrée 1914 : par ordre alphabétique, Charles Barrois, Charles-Eugène Bertrand, Auguste Boulanger, Alphonse Buisine, Benoît-Côme Damien, Gustave Demartres, Paul Hallez, Alphonse Malaquin, René Paillot, Henri Pélabon, Albert Petot, René Swyngedauw. Ils sont vingt et un avant la guerre. Les mobilisés sont Paul Bertrand, Jean Chazy, Jean Clairin, Richard Fosse, Jacques de Lapparent, Paul Lemoult, Héloïse Ollivier et Paul Pascal. Quatre chefs de travaux et préparateurs sont présents sur vingt et un et cinq garçons de laboratoire sur quinze auparavant.

En physique, Benoît-Côme Damien et René Paillot organisent les cours et préparent les certificats PCN et SPCN. En sus, Damien encadre un doctorant et une aspirante à l'agrégation. En 1915, René-Eugène Roberget, professeur agrégé de physique du lycée de Douai, renforce le personnel et est chargé des fonctions de chef de travaux de physique. Un cours de physique industrielle subsiste grâce à l'assistance de « bénévoles », la majorité des étudiants étant mobilisés. Les bénévoles sont des auditeurs libres dans le langage de l'époque, soit des personnes du métier intéressées par le cours public mais qui ne visent pas le passage des examens. Après l'incendie de l'Institut d'électrotechnique, René Swyngedauw enseigne à l'Institut de physique où il restaure également les appareils d'électrotechnique sauvés. Rapidement, il préconise « la construction d'un laboratoire provisoire pour le service sinistré » et demande un crédit au Parlement pour « l'édification d'un nouvel institut proportionné aux besoins considérables qui vont surgir $\gg^{30}$, crédit qu' il n'obtiendra pas.

En mathématiques générales, Gustave Demartres encadre les cours suivis par sept étudiants en 1914-191s et prépare un élève au certificat de calcul différentiel et intégral. Une étudiante, Mademoiselle Merchier, réalise une conférence complémentaire pour les élèves en difficultést. Au début de l'année 1915, la classe de mathématiques spéciales du lycée Faidherbe est accueillie à la Faculté des sciences. L'enseignement des mathématiques générales prend alors une grande importance avec trente-deux étudiants, parmi lesquels trois bénévoles.

29.- Béatrice TOUCHELAY, « Les monnaies de nécessité ou la grande débrouille dans le Nord occupé (1914-1918) 》, Colloque international Recherche et régulation sur « La théorie de la régulation à l'épreuve des crises », Paris, 10-12 juin 2015, 10 p.

30.- Alphonse MALAQUIN, « Rapport sur la situation de l'enseignement supérieur à Lille pendant l'année scolaire 1915-1916», dans Annales de l'Université de Lille : rapport annuel $d u$ Conseil de l'Université..., Lille, Imprimerie Le Bigot, p. 62.

31.- Benoit Côme DAMIEN, « Faculté des sciences : Rapport sur la situation et les travaux de la Faculté pendant l'année scolaire 1914-1915 », dans Annales de l'Université de Lille: rapport annuel du Conseil de l'Université..., Lille, Imprimerie Le Bigot, p. 30. 
Henri Deroide, professeur agrégé de mathématiques au lycée Faidherbe, enseigne également à la Faculté durant la guerre, avant d'être mobilisé. En son absence, Charles Vallée de l'École des Arts et Métiers de Lille le remplace. Les cours de mécanique sont assurés par Albert Petot et Auguste Boulanger. Ce dernier, maître de conférences en mécanique, est nommé au Conservatoire des Arts et Métiers de Paris en 1914 mais ne réussit pas à sortir de Lille avant 1915. Pour rejoindre son poste, il traverse la Belgique à pied aidé de passeurs, puis les Pays-Bas, avant d'embarquer à destination de Londres pour rejoindre enfin Paris. Quelques mois plus tard, sa femme et ses enfants le rejoignent à Versailles par le train, et ce en passant par l'Alsace et la Suisse. Après son départ, Albert Petot poursuit l'enseignement de mécanique devant six étudiant ${ }^{32}$.

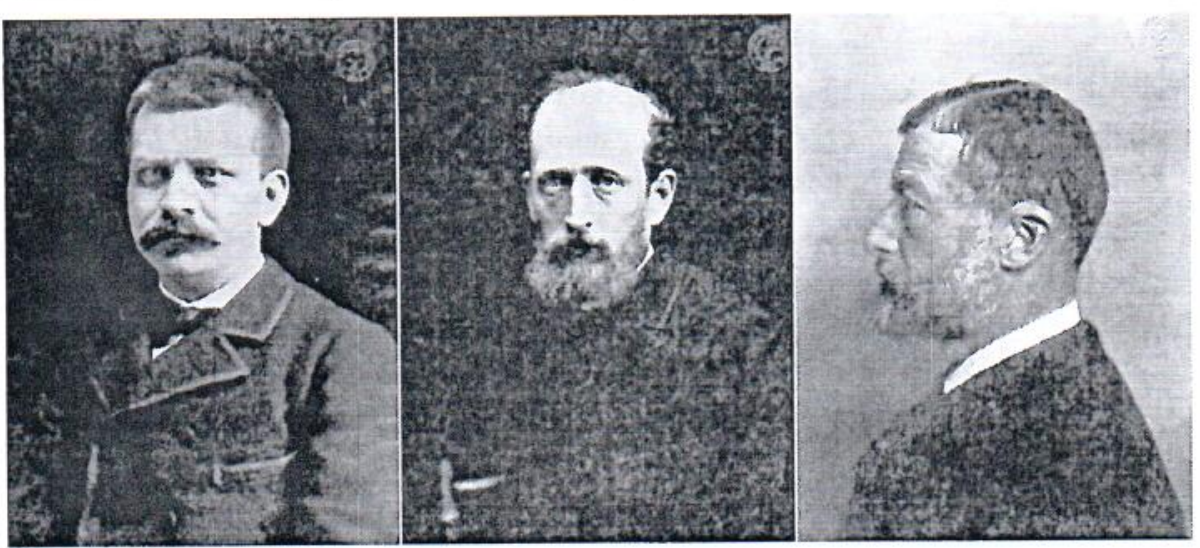

Figure 9: À gauche, Benoît-Côme Damien (Bibliothèque municipale de Lille, Fonds Lefebvre 5, 35, fin XIX' siècle) ; au centre, Gustave Léon Demartres (Gustave Léon Demartres, Bibliothèque municipale de Lille, Fonds Lefebvre 5, 45); à droite, Charles Barrois (Bibliothèque municipale de Lille, Fonds Lefebvre 5, 8).

En chimie, Alphonse Buisine et Henri Pélabon préparent les étudiants aux certificars PCN et SPCN. Fin décembre 1917, des professeurs de l'Université de Lille et leurs épouses sont pris en otage. Le 6 janvier 1918, Alphonse Buisine est déporté ainsi que son collègue Mouchet, professeur de droit, en Lituanie, tandis que son épouse et Mesdames Calmette et Lemoine, épouses de professeurs de médecine, partent pour le camp d'Holzminden en Allemagne ${ }^{33}$. De santé « plus que précaire », Buisine meurt fin mars au camp de Milejgany. Pélabon remplace Buisine à la direction de l'École de chimie, aidé par M. de Saint-Étienne, chef de travaux, et Pierre-Léon Perrier, préparateur. En l'absence de Paul Pascal, maître de conférences, et d'Auguste Boulanger, chargé d'un cours libre, Paul Émile Guenez, chimiste en chef au laboratoire du ministère

32.- Ibid., rapport sur l'année 1916-1917, p. I26.

33.- Jules JACQUEY, « Rapport sur la situation de l'enseignement supérieur à Lille pendant l'année scolaire 1917-1918 » dans Annales de l'Université de Lille : rapport annuel du Conseil de l'Université..., Lille, Imprimerie Le Bigot, p. 144. des Finances à L colorants, le sucre

En zoologie, de zoologie, de ? l'entretien des co. naturelle grâce à li: des travaux pratic la diminution de remplacement $\ll \hat{c}$ alors privilégiés ${ }^{36}$. présenter régulièrı aller régulièremen Ce système des no Paul Hallez n'a qr maritime du Porte Bertrand est seul locaux, quelques ét Il décède le I8 aoû bombardement du dès le 20 août et co En géologie et mir et encadre en SPC géologie et de paléc est secondé par les ، durant l'occupation de minéralogie et $\mathrm{C}$ classement des coll

Les auditeurs p Durant l'année univ compte 278 étudian

34.- Benoît Côme DAN Faculté pendant l'a annuel du Conseil a

35.- Ibid., « rapport su. I9I5 » dans Annales Imprimerie Le Bigc

36.- Benoît Côme DAM Faculté pendant l'a annuel du Conseil d

37.- Jean-François CON l'bistoire, Lille, Pres

38.- Jules DEROCQUIC pendant l'année scc du Conseil de l'Uniu

39.- Benoît Côme DAM Faculté pendant l'a: annuel du Conseil as 
des Finances à Lille, donne des cours sur les matériaux de construction, les colorants, le sucre ou encore le lait ${ }^{34}$.

En zoologie, Alphonse Malaquin encadre les étudiants du certificat de zoologie, de PCN et de SPCN. Il assure également la conservation et l'entretien des collections d'entomologie à la Faculté et au musée d'Histoire naturelle grâce à la présence d'un gardien et d'une gardienne ${ }^{35}$. L'enseignement des travaux pratiques est contrarić par l'absence d'animaux de laboratoire et la diminution de réserves d'animaux destinés aux dissections. Des animaux de remplacement « autour de soi » et les travaux d'anatomie microscopique sont alors privilégiés ${ }^{36}$. Malaquin et son épouse font partie des otages qui doivent se présenter régulièrement à la Kommandantur pour attester de leur présence et aller régulièrement dormir à la citadelle sous surveillance militaire allemande. Ce système des notables otages semble perdurer tout au long de l'occupation ${ }^{37}$. Paul Hallez n'a quant à lui pu assurer depuis Lille la direction du laboratoire maritime du Portel situé dans la zone française. En botanique, Charles-Eugène Bertrand est seul au service du laboratoire. Il enseigne en PCN et, faute de locaux, quelques étudiants viennent chez lui préparer le certificat de botanique. Il décède le 18 août 1917 . Son domicile lillois a par ailleurs été détruit lors du bombardement du is août. Henri Fockeu, professeur de médecine, le supplée dès le 20 août et continuera à enseigner à la Faculté des sciences après la guerre ${ }^{38}$. En géologie et minéralogie, Charles Barrois assure le cours général et appliqué et encadre en SPCN. Faute de déplacement possible sur le terrain, les cours de géologie et de paléontologie ont lieu dans les galeries du musée houiller. Barrois est secondé par les étudiants Pierre Pruvost, qui soutiendra la seule thèse lilloise durant l'occupation en mai 1918, pour des conférences et des travaux pratiques de minéralogie et Georges Dubois, futur docteur de la Faculté en 1924, pour le classement des collections ${ }^{39}$.

\section{Les auditeurs présents aux cours de la faculté des sciences}

Durant l'année universitaire 1913-1914 qui précède la guerre, la faculté des sciences compte 278 étudiants : 97 préparent un des dix-sept CES, 6 l'agrégation, 46 un

34- - Benoît Côme DAMIEN, « Faculté des sciences: Rapport sur la situation et les travaux de la Faculté pendant l'année scolaire 1917-1918 », dans Annales de l'Université de Lille : rapport annuel du Conseil de l'Université..., Lille, Imprimerie Le Bigot, p. 178.

35. - Ibid., « rapport sur la situation et les travaux de la Faculté pendant l'année scolaire 1914I915 » dans Annales de l'Université de Lille : rapport annuel du Conseil de l'Université..., Lille, Imprimerie Le Bigot, p. 32.

36.- Benoît Côme DAMIEN, « Faculté des sciences : Rapport sur la situation et les travaux de la Faculté pendant l'année scolaire 1916-1917 », dans Annales de l'Université de Lille : rapport annuel du Conseil de l'Université..., Lille, Imprimerie Le Bigot, p. 128-129.

37.- Jean-François CONDETTE, La faculté des Lettres de Lille de 1887 à 1945. Une faculté dans l'histoire, Lille, Presses Universitaires du Septentrion, 1999, p. $231-232$.

38.- Jules DEROCQUIGNY, « Rapport sur la situation de l'Université de Lille : rapport annuel pendant l'année scolaire 1916-1917 》, dans Annales de l' Universi

du Conseil de l'Université..., Lille, Imprimes sciences: Rapport sur la situation et les travaux de la

39.- Benoît Côme DAMIEN, « Faculte des sciences dans Annales de l'Université de Lille : rapport Faculté pendant l'annél déniversité..., Lille, Imprimerie Le Bigot, p. 34. 
certificat PCN, Ir 6 un diplôme universitaire (DU), 8 le professorat de sciences appliquées et 5 sont des bénévoles. Parmi eux, 6I sont de nationalité étrangère : 54 préparent le diplôme d'ingénieur électricien, dont 40 Russes, 5 le diplôme d'ingénieur chimiste et 2 élèves originaires des Pays-Bas sont en « recherche de laboratoire ». Parmi les résultats de l'année universitaire 1913-1914 : un étudiant est reçu à l'agrégation de mathématiques, deux jeunes filles à l'agrégation des filles, un étudiant au professorat des écoles primaires supérieures en sciences appliquées, une jeune fille au certificat d'aptitude à l'enseignement secondaire des jeunes filles, une jeune fille est admise à l'École normale primaire supérieure de Fontenay-aux-Roses.

L'année 1914-1915 est quant à elle désastreuse. Du fait de l'occupation de la ville par l'armée allemande, aucune session d'examen n'a lieu en octobre et novembre I9I 4 comme le souligne un extrait des Annales de l'Université de Lille:

Il n'a été fait aucun acte scolaire, en dehors d'un examen pour le certificat de botanique [du fait d'une scolarité antérieure]. Nous avons insisté plus haut sur le labeur considérable, qu'ont fourni les membres de la Faculté en n'ayant pour élèves que des auditeurs bénévoles. L'unique session de baccalauréat du mois d'avril a compté quatre candidats à la première partie, trois à la seconde. Tous ont été reçus ${ }^{+0}$.

Les effectifs se reconstituent peu à peu. En 1915-1916, il y a cinquante-six étudiants. En 1916-1917, ils sont quatre-vingt-seize. Ils sont 163 en 1917-1918. Les auditeurs sont des garçons non mobilisés, des filles et des bénévoles. Les filles apparaissent avant la guerre dans différents CES, toutefois le baccalauréat et les lycées de jeunes filles ne seront vraiment à leur portée qu'après l'identification des enseignements masculins et féminins en 1924. Jusqu'alors, elles accèdent aux études universitaires par des voies singulières et se cantonnent dans les seules voies de l'enseignement et de l'administration. Mais en 1915-1916 à Lille, «Pour la première fois, des élèves jeunes filles préparent le diplôme d'ingénieur chimiste : c'est une expérience qu'il sera intéressant de suivre ${ }^{41} \gg$. Ainsi, quatre élèves dont deux jeunes filles sont inscrits en première année, cinq élèves en deuxième année et trois étudiants suivent le diplôme de chimie appliquée. Quelques bénévoles se joignent à eux ${ }^{42}$. Les bénévoles sont des auditeurs libres qui assistent aux cours mais qui ne se présentent pas aux examens et ne prétendent pas aux grades. Dans le CES de Physique industrielle, les bénévoles sont des ingénieurs dans l'industrie électrique de construction et d'exploitation. En géologie ce sont des habitants de quartier, des membres de sociétés savantes lilloises comme La Société des amis de Lille.

40.- Paul CHARMEIL, « Rapport sur la situation de l'enseignement supérieur à Lille pendant l'année scolaire 1914-1915 », dans Annales de l'Université de Lille : rapport annuel du Conseil de l'Université..., Lille, Imprimerie Le Bigot, p. II.

4I.- Benoît Côme DAMIEN, « Faculté des sciences : Rapport sur la situation et les travaux de la Faculté pendant l'année scolaire 1916-1917 », dans Annales de l'Université de Lille : rapport annuel du Conseil de l'Université..., Lille, Imprimerie Le Bigot, p. 128. 42.-Ibid., p. 128.

Ces étudiant en atteste une $\mathrm{l}$ matiques généra Demartres décé: mathématiques 1

« Lille, le 4

Mon cher $\mathrm{C}$

Le 28 nov

M. Chapelc

Docteur ave

M. Milloux pation allem: parisien, pré de lui penda:

Nous savons cours habitu teurs, et en jours de la g plus élément qui put s'adr Cet enseigne d'entrer pres Navale, à l'É quinze ans en tiques. Même en ces tristes réalisé par De auraient été cl M. Borel, qui il $y$ a trente Différentiel e de Milloux $\mathrm{q}$ des étudiants sentant la Fac du Professeur successeur auj personne : il $\mathrm{v}$ jours sombres ont été animés

43.- Université de Iill Chazy. 
Ces étudiants et étudiantes bénéficient d'un enseignement de qualité comme en atteste une lettre touchante de Jean Chazy, titulaire de la chaire de mathématiques générales à Lille de I9II à 1933, qui met en valeur le rôle de Gustave Demartres décédé en 1919. Celui-ci a porté à bout de bras l'enseignement des mathématiques pendant la guerre et meurt peu après.

« Lille, le 4 décembre 1924.

Mon cher Doyen,

Le 28 novembre dernier, M. Henri Milloux, qui cette année supplée M. Chapelon parmi nous, a soutenu sa thèse en Sorbonne, et a été reçu Docteur avec la mention très honorable. En souvenir du dévouement que M. Milloux a rencontré chez les Professeurs de notre Faculté pendant l'occupation allemande et surtout chez notre regretté collègue Demartres, le jury parisien, présidé par M. Borel, m'a fait l'honneur de m'inviter à siéger à côté de lui pendant cette soutenance de thèse.

Nous savons tous comment pendant la guerre Demartres n'a pas continué ses cours habituels d'Analyse et de Géométrie qui auraient eu trop peu d'auditeurs, et en l'absence de Clairin mobilisé et d'ailleurs tué dès les premiers jours de la guerre, comment Demartres a pris la charge d'un enseignement plus élémentaire de Mathématiques générales et de Mathématiques spéciales qui put s'adresser au plus grand nombre possible d'étudiants et d'étudiantes. Cet enseignement a permis notamment à un certain nombre de jeunes gens d'entrer presque de suite à la libération à l'École Polytechnique, à l'École Navale, à l'École des Postes et Télégraphes, et a permis à Milloux, qui avait quinze ans en 1914 de posséder en 1919 les certificats de la licence de mathématiques. Même en faisant abstraction de l'effet moral d'un bel exemple d'énergie en ces tristes circonstances, l'on peut dire que, sans l'effort d'enseignement réalisé par Demartres, la carrière et la vie de Milloux et de tous ces jeunes gens, auraient été changées, retardées, presque sûrement diminuées.

M. Borel, qui lui-même était ici Maître de Conférences de Mathématiques il y a trente années, alors que Demartres était déjà Professeur de Calcul Différentiel et Intégral, a rappelé publiquement à la soutenance de thèse de Milloux que notre ancien Collègue a fait pendant la guerre à l'égard des étudiants plus que son devoir. J'ai été fier d'être associé comme représentant la Faculté des Sciences de Lille à cet hommage rendu à la mémoire du Professeur dont j'ai été le dernier maître de conférences et dont je suis le successeur aujourd' hui. Mais l'hommage qui m'a été fait ne s'attache pas à ma personne : il va à chacun de mes collègues, et surtout à ceux qui, pendant les jours sombres de l'occupation aux côtés de Demartres et du Doyen Damien, ont été animés du même dévouement. »

\section{Chazy $^{+3}$}

43.- Université de Lille, Sciences et Technologies, dossier administratif individuel de Jean Chazy. 
Henri Milloux est reçu en mathématiques générales à Lille en juin 1917 et obtient la licence avec calcul différentiel dans les certificats requis en juillet 1918. Mathématicien remarquable, il sera élu membre de l'Académie des Sciences en 1959.

Le passage des examens et du baccalauréat

La progression du nombre d'élèves et de certifiés est constante sur les quatre années de guerre, bien que le nombre de sessions varie selon les années. Pourl'année scolaire 1917-1918, soixante-et-onze CES sont délivrés pour cent vingt-deux candidats ; douze licences complètes ainsi qu' un DES obtenu par Pierre-Léon Perrier, préparateur de chimie, sont délivrés alors qu'une thèse est soutenue par Pierre Pruvost en géologie ${ }^{44}$. Parfois, des élèves sont évacués par l'autorité allemande comme en 1916 quand un élève de physique industrielle n'a pas pu rentrer dans la salle pour passer son examen. Les tableaux suivants donnent le nombre d'étudiants inscrits dans les différents CES suivant les années d'occupation.

Les étudiants inscrits dans les différents certificats d'études supérieures de la licence à la faculté des sciences de Lille.

\begin{tabular}{|c|c|c|c|c|c|c|c|c|}
\hline$\underset{\widetilde{\Xi}}{\tilde{\Xi}}$ & $\begin{array}{l}Z \\
\mathcal{U}\end{array}$ & 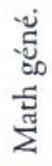 & 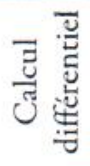 & 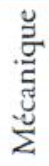 & 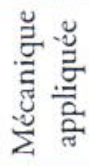 & 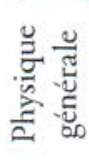 & 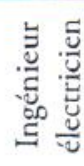 & 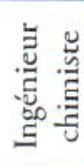 \\
\hline $1913 / 14$ & 46 & 37 & 9 & 8 & IO & 29 & 94 & 39 \\
\hline $1914 / 15$ & 7 & 0 & $\circ$ & $\circ$ & $\circ$ & $\circ$ & 0 & o \\
\hline $1915 / 16$ & 21 & 7 & I & 4 & 0 & 3 & 2 & 0 \\
\hline $1916 / 17$ & 12 & 24 & 0 & 4 & 2 & 13 & 6 & 9 \\
\hline $1917 / 18$ & $2 \mathrm{I}$ & 37 & I & I2 & I & 20 & 3 & 24 \\
\hline $1918 / 19$ & 58 & 19 & I & 3 & 4 & II & I & 38 \\
\hline
\end{tabular}

\begin{tabular}{|c|c|c|c|c|c|c|c|}
\hline 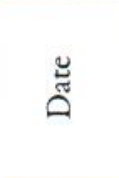 & $\begin{array}{l}\frac{0}{80} \\
0 \\
0 \\
0\end{array}$ & $\begin{array}{l}Z \\
\vdots \\
\hbar\end{array}$ & 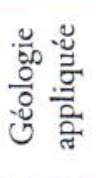 & 苞 & 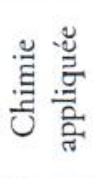 & 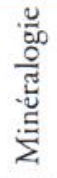 & 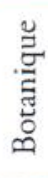 \\
\hline $1913 / 14$ & 8 & 18 & 8 & 14 & 4 & 22 & 3 \\
\hline $1914 / 15$ & 0 & 0 & 0 & 0 & 0 & 0 & 1 \\
\hline $1915 / 16$ & I & 6 & I & 0 & 0 & 0 & 0 \\
\hline $1916 / 17$ & 6 & 9 & 0 & IS & 7 & 3 & 2 \\
\hline $1917 / 18$ & 4 & 13 & 2 & 25 & 7 & 4 & 1 \\
\hline $1918 / 19$ & 58 & 19 & I & 3 & 4 & II & 3 \\
\hline
\end{tabular}

44.- Pierre PRUVOST, Préparateur au Musée Houiller de l'Université de Lille, Thèses présentées à la Faculté des Sciences de l'Université de Lille pour obtenir le grade de docteur ès sciences naturelles le 18 mai I9I8. Première thèse: Introduction à l'Étude du terrain houiller du Nord et du Pas-de-Calais : La Faune continentale du terrain houiller du Nord et du Pas-deCalais. Seconde thèse : Propositions données par la Faculté. MM Ch. Barrois : Président, A. Malaquin et H. Fockeu : examinateurs, Paris, Imprimerie Nationale, 1919.

partie : s

sont exa! niveau d avant lec des exam avancée ! erudiants

dans une partir d'o Lille est i: laissez-pa: a Tourcoi lettres, co:

Les effect: sciences d

Date

\begin{tabular}{l}
\hline $10 / 1913$ \\
\hline $0-1914$ \\
\hline $10 / 2014$ \\
\hline $0+1915$ \\
\hline $10 / 1915$ \\
\hline $11 / 1915$ \\
\hline $01 / 1916$ \\
\hline $0-1916$ \\
\hline $10 / 1916$ \\
\hline $0+1917$ \\
\hline $06 / 1917$ \\
\hline $0-/ 1917$ \\
\hline $10 / 191-$ \\
\hline $0-1918$ \\
\hline $12 / 1918$ \\
\hline $0+1919$ \\
\hline $5-$ Jules JAC \\
l'annee sc \\
del'Unise
\end{tabular}


L'organisation des épreuves du baccalauréat est une préoccupation constante et majeure. Comme avant la guerre, les candidats au baccalauréat « Première partie : sciences et langues vivantes » et «Deuxième partie : mathématiques 》 sont examinés à la faculté des sciences. Le nombre de sessions annuelles varie. Le niveau d'exigence est maintenu et le taux de réussite est de l'ordre de ce qu'il était avant le déclenchement de la guerre. Les événements portent préjudice au passage des examens. Il n'y a pas de session en octobre 1914. En juin 1917, une session est avancée par le recteur Georges Lyon après qu'un ordre ait été donné à certains étudiants et élèves du second degré de subir un examen médical pour aller travailler dans une autre région. En définitive, l'évacuation de ces jeunes gens sera annulée. À partir d'octobre 1917 , la circulation est interdite entre Lille et Roubaix-Tourcoing. Lille est isolée et les examens sont gravement compromis. En avril et mai 1918, un laissez-passer est délivré à certains professeurs de la faculté. Il leur permet d'aller à Tourcoing pour faire passer le baccalauréat et les examens de sciences, droit et lettres, contrairement aux usages, tandis qu'une autre session se déroule à Lille ${ }^{45}$.

Les effectifs de candidats et de reçus aux sessions de baccalauréat de la faculté des sciences de Lille (1913-1920).

\begin{tabular}{|c|c|c|c|c|c|c|c|c|c|}
\hline \multirow[b]{2}{*}{ Date } & \multicolumn{3}{|c|}{$\begin{array}{c}\mathbf{I}^{\mathrm{re}} \text { partie «Sciences et } \\
\text { langues vivantes » }\end{array}$} & \multicolumn{3}{|c|}{$\begin{array}{c}2^{\mathrm{c}} \text { partie } \\
\text { «Mathématiques 》 }\end{array}$} & \multicolumn{3}{|c|}{ Total } \\
\hline & 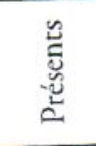 & 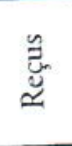 & $\frac{\mathscr{\mathscr { U }}}{\tilde{J}}$ & 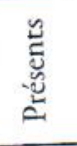 & 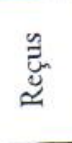 & $\frac{\stackrel{\mathscr{U}}{\tilde{Z}}}{\mathscr{Z}}$ & 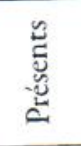 & $\underset{\mathscr{Z}}{\mathscr{Z}}$ & $\frac{\mathscr{2}}{\widetilde{\mathscr{Z}}}$ \\
\hline $10 / 1913$ & 117 & 54 & 63 & 77 & 34 & 43 & 194 & 88 & 106 \\
\hline $07 / 1914$ & 271 & 109 & 162 & 176 & 106 & 70 & 447 & 215 & 232 \\
\hline $10 / 2014$ & 0 & 0 & 0 & $\circ$ & 0 & 0 & 0 & 0 & $\circ$ \\
\hline $04 / 1915$ & 4 & 4 & 0 & 3 & 3 & 0 & 7 & 7 & 0 \\
\hline $10 / 1915$ & 21 & 9 & 12 & 18 & 13 & 5 & 39 & 22 & 17 \\
\hline 11/I9IS & 4 & 3 & I & 8 & 7 & I & 12 & 10 & 2 \\
\hline $01 / 1916$ & 7 & 4 & 3 & 4 & 4 & $\circ$ & II & 8 & 3 \\
\hline $07 / 1916$ & 33 & 18 & 15 & 25 & 18 & 7 & 58 & 36 & 22 \\
\hline $10 / 1916$ & $2 \mathrm{I}$ & 9 & $\mathrm{I} 2$ & II & 8 & 3 & 32 & 17 & 15 \\
\hline $04 / 1917$ & 2 & 0 & 2 & 4 & 4 & $\circ$ & 6 & 4 & 2 \\
\hline $06 / 1917$ & 31 & 16 & 15 & 47 & 39 & 8 & 78 & 55 & 23 \\
\hline $07 / 1917$ & 13 & 8 & 5 & 10 & 9 & I & 23 & 17 & 6 \\
\hline $10 / 1917$ & 24 & 12 & 12 & 9 & 7 & 2 & 33 & 19 & 14 \\
\hline $07 / 1918$ & $5 \mathrm{I}$ & 23 & 28 & 47 & 30 & 17 & 98 & 53 & 45 \\
\hline $12 / 1918$ & 17 & II & 6 & 19 & 18 & $\mathrm{I}$ & 36 & 29 & 7 \\
\hline $04 / 1919$ & 10 & 10 & $\circ$ & 10 & 10 & 0 & 20 & 20 & $\circ$ \\
\hline
\end{tabular}

45.- Jules JACQUEY, « Rapport sur la situation de l'enseignement supérieur à Lille pendant l'année scolaire 1917-1918 》, dans Annales de l'Université de Lille: rapport annuel du Conseil de l'Université..., Lille, Imprimerie Le Bigot, p. 146-147. 


\begin{tabular}{|c|c|c|c|c|c|c|c|c|c|}
\hline \multirow[b]{2}{*}{ Date } & \multicolumn{3}{|c|}{$\begin{array}{c}\mathrm{I}^{\mathrm{re}} \text { partie « Sciences et } \\
\text { langues vivantes }\end{array}$} & \multicolumn{3}{|c|}{$\begin{array}{c}2^{\mathrm{c}} \text { partie } \\
\text { «Mathématiques » }\end{array}$} & \multicolumn{3}{|c|}{ Total } \\
\hline & 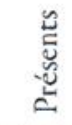 & $\stackrel{\mathscr{U}}{\mathscr{Z}}$ & 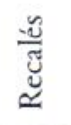 & 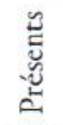 & 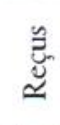 & $\frac{\ddot{u}}{\tilde{U}}$ & 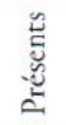 & $\stackrel{\tilde{Z}}{\mathscr{Z}}$ & $\frac{\mathscr{U}}{\tilde{\mathscr{U}}}$ \\
\hline $07 / 1919$ & 140 & 6I & 79 & 165 & 95 & 70 & 305 & 156 & 149 \\
\hline $10 / 1919$ & 80 & 42 & 38 & 74 & 43 & $3 \mathrm{I}$ & 154 & 85 & 69 \\
\hline $01 / 1920$ & 4 & 4 & 0 & 6 & 6 & 0 & 10 & 10 & ० \\
\hline $03 / 1920$ & I & I & 0 & 3 & 3 & 0 & 4 & 4 & 0 \\
\hline $07 / 1920$ & 200 & 86 & $\mathrm{II}_{4}$ & 202 & 142 & 60 & 402 & 228 & 174 \\
\hline
\end{tabular}

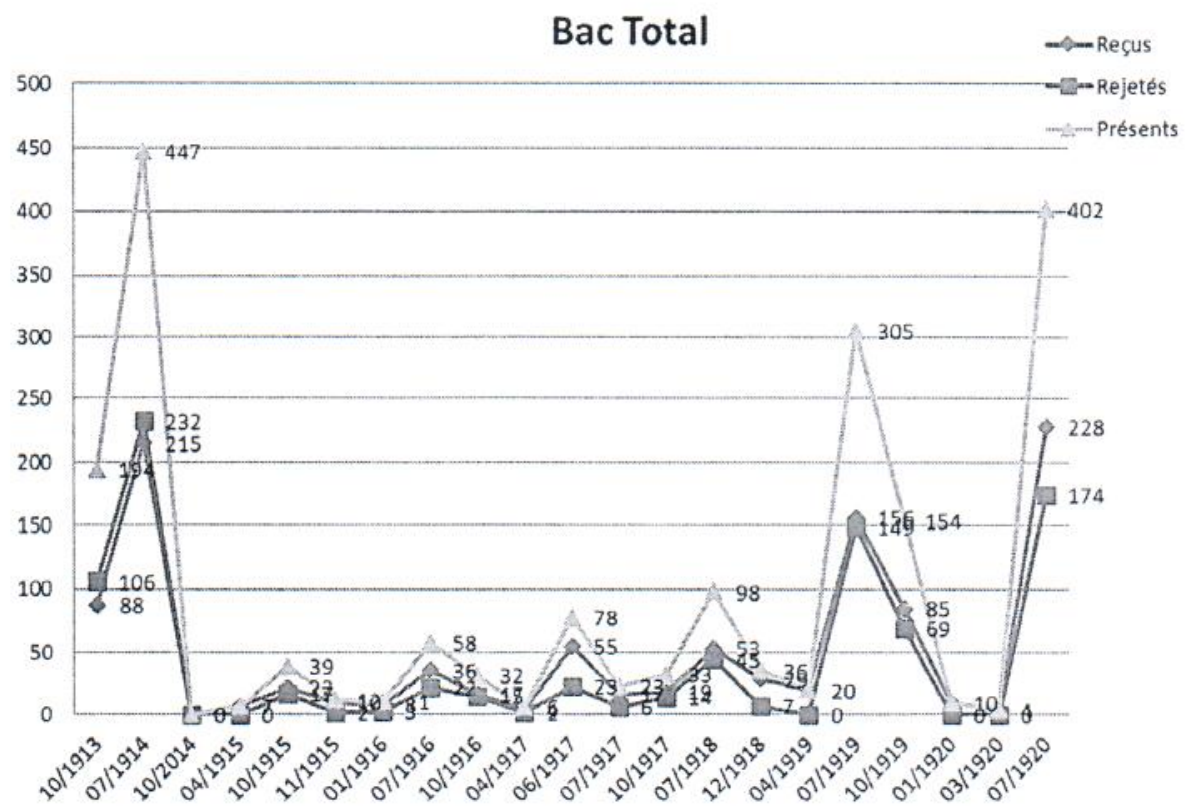

\section{L'apport de la faculté des sciences à la vie scientifique}

Dans un tel contexte marqué par les réquisitions allemandes, par la vie très difficile et par la proximité du front, une activité scientifique de recherche est cependant maintenue à la faculté.

\section{Les recherches universitaires pendant l'occupation}

Dans les laboratoires, des recherches se poursuivent pendant la période de l'occupation bien qu'elles soient plus difficiles à mener. En mécanique, Albert Petot continue ses travaux sur la dynamique de l'automobile (embrayages, changements de vitesse, transmissions par cardan, mécanismes et conditions de stabilité dans les courbes et les pentes) sans pouvoir réaliser les expériences souhaitées. Il met ses notes au propre « pour qu'elles puissent, même incomplètes, être utilisées

par ceux de se industrielle, $R$, triphasés, cous les pouvoirs tt En zoologie, $t$ campagne, sur Portel étant pe est dans l'imp publier ses rect l'activité conti Charles Barroi sera soutenue li universitaire so

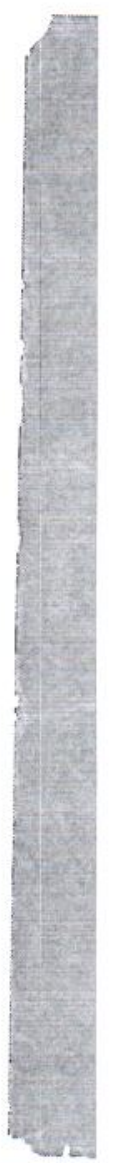

Figure et imp: Évo-É

46.- Benoît Côme Faculté pend: annuel du Co? 
par ceux de ses lecteurs qui voudraient continuer ses recherches ${ }^{46}$. En physique industrielle, René Swyngedauw travaille sur l'objet de ses cours (câbles électriques triphasés, court-circuit des alternateurs). En chimie, Henri Pelabon travaille sur les pouvoirs thermoélectriques des alliages au cours de l'année scolaire 1915-1916. En zoologie, Alphonse Malaquin fait des recherches, sans observation en pleine campagne, sur quelques parasites de plantes cultivées. Les cultures biologiques du Portel ćtant perdues, faute de personnel, et Paul Hallez se trouvant à Lille, celui-ci est dans l'impossibilité de communiquer avec son laboratoire et avec Paris pour publier ses recherches. En botanique, l'activité du laboratoire est nulle. En géologie, l'activité continue au minimum et ce avec la thèse de Pierre Pruvost, assistant de Charles Barrois, sur l'étude du terrain houiller du Nord et du Pas-de-Calais, qui sera soutenue le I 8 mai 1918 à Lille pendant la guerre. Ce sera d'ailleurs la seule thèse universitaire soutenue à la Faculté des Sciences durant l'occupation allemande.

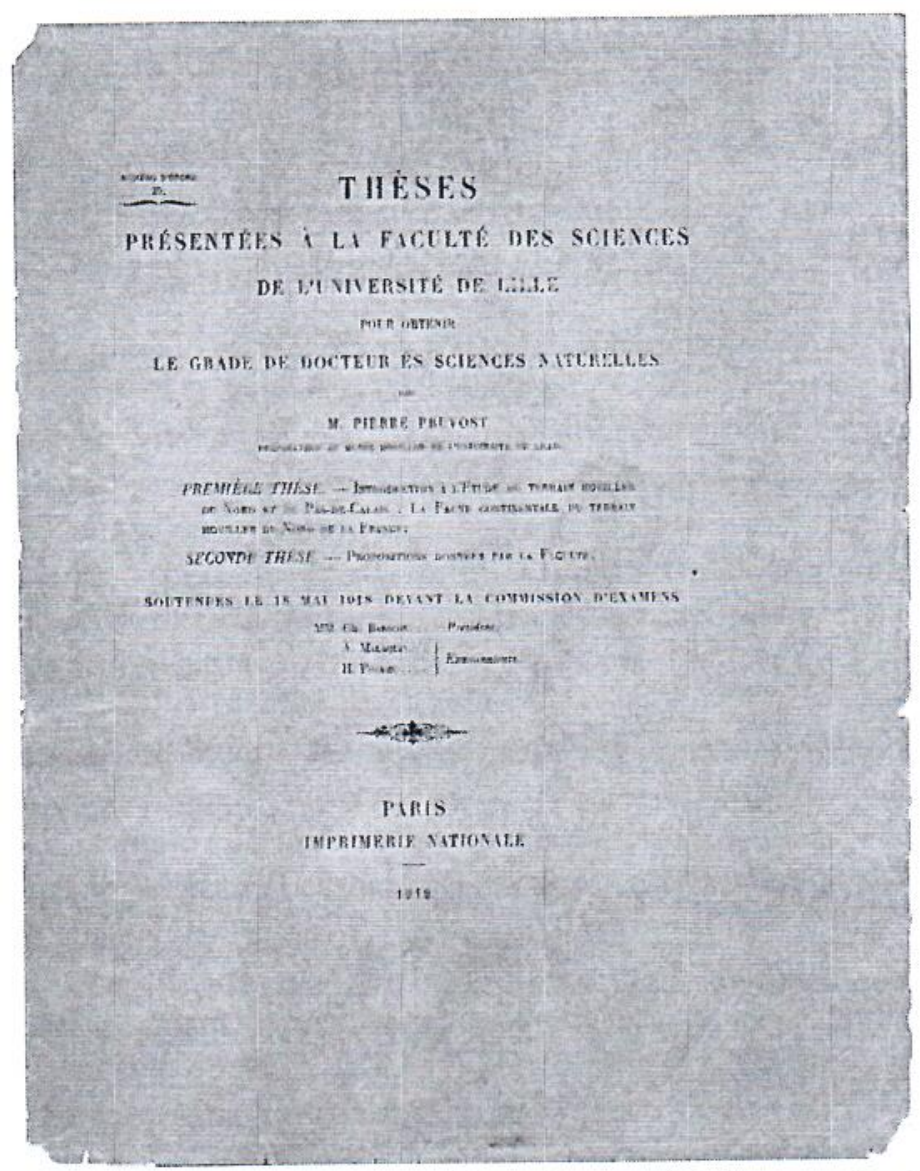

Figure 10: Thèse présentée par Pierre Pruvost à Lille en mai 1918 et imprimée à Paris en 1919 (Bibliothèque recherche, UMR 8198 Évo-Éco-Paléo, Université de Lille, Sciences et Technologies).

46.- Benoît Côme DAMIEN, « Faculté des sciences : Rapport sur la situation et les travaux de la Faculté pendant l'année scolaire 1915-1916 », dans Annales de l'Université de Lille : rapport annuel du Conseil de l'Université..., Lille, Imprimerie Le Bigot, p. 8I. 
Les publications des recherches, menées à Lille et dans ses environs, sont stoppées de manière générale en raison de la séparation avec la zone non occupée qui vient interrompre le processus habituel de publication. Il en va de même pour les publications des sociétés savantes dont l'activité se poursuit tout de même pour certaines, comme la Société géologique du Nord.

\section{L'apport des scientifiques pendant la guerre à Lille et en France}

La Grande Guerre est une guerre « scientifique ». Jamais les scientifiques n'ont été autant sollicités et impliqués. Le Manifeste des 93 ou Appel des intellectuels allemands aux nations civilisées du 4 octobre 1914 donne la mesure de l'engagement des savants pour soutenir l'Allemagne guerrière, en réponse à l'indignation internationale devant l'invasion de la Belgique et l'incendie de l'une des plus anciennes universités d'Europe, à savoir l'Université de Louvain, sa bibliothèque et ses archives. En effet, la presse relaie la qualification « acte de barbarie » utilisée par les intellectuels anglais et américains en particulier. Parmi les signataires du manifeste, le mathématicien Félix Klein, les physiciens Max Planck et Wilhelm Röntgen, le chimiste Fritz Haber sont à citer. Ce dernier est le père des gaz de combat. Ces gaz asphyxiants à base de chlore sont utilisés début ig1s au nord d'Ypres. Son épouse, chimiste éminente, s'élève contre ses recherches et se suicide en 1915 . Haber recevra le Prix Nobel de chimie en 1918 pour la synthèse de l'ammoniac, composant essentiel des engrais et des explosifs. Albert Einstein refuse de signer le Manifeste des 93. Ce manifeste cautionnant les faits de guerre et d'occupation allemands sera en partie la cause de l'exclusion des chercheurs allemands et de langue allemande de la scène scientifique internationale au lendemain de la guerre ${ }^{47}$.

Au-delà des recherches réalisées dans le cadre universitaire, les scientifiques lillois apportent leurs compétences à l'effort de guerre, œuvrant ainsi à la Défense nationale. En 1915, Paul Bertrand, affecté à la médecine militaire à Amiens, travaille sur le typhus. En 1915-1916, Alphonse Buisine, chimiste, est chargé par la municipalité lilloise d'examiner des échantillons de farine fournie à la ville par l'autorité allemande ${ }^{+8}$. En 1916, Paul Lemoult, chimiste mobilisé depuis 1914, est nommé directeur de l'usine Vandier \& Despret à La Pallice, près de La Rochelle. L'usine est créée par Jacques Vandier, son usine lilloise étant dans la zone d'occupation allemande. Il n'est ainsi pas étonnant que ce soit un chimiste lillois qui ait été mis à la direction. L'usine est spécialisée dans la fabrication de la mélinite et de l'acide picrique qui sont des constituants explosifs utilisés pour la conception des obus. Elle produit dix-sept tonnes par jour de produits particulièrement dangereux destinés aux besoins de l'armée. Malheureusement, l'usine explose le $\mathrm{I}^{\mathrm{er}}$ mai 1916. Cent trente-huit blessés et cent soixante-seize morts sont à déplorer, parmi lesquels Paul Lemoult et Victor Sanson. Celui-ci était préparateur en chimie générale à Lille depuis 1900 et avait rejoint « son maître » lors de sa nomination

47.- Roswitha REINBOTHE, « L'exclusion des scientifiques allemands et de la langue allemande des congrès scientifiques internationaux après la Première Guerre mondiale », Revue germanique internationale, 12, 2010, p. 193-208.

48.- Benoît Côme DAMIEN, « Faculté des sciences: Rapport sur la situation et les travaux de la Faculté pendant l'année scolaire 1914-1915 », dans Annales de l'Université de Lille : rapport annuel du Conseil de l'Université..., Lille, Imprimerie Le Bigot, p. 31. 
ذ̇ ta tête de l'usine de La Pallice. En 1919, Jean Chazy, mathématicien, est distingué al'Ordre de l'artillerie en tant qu' « officier savant et courageux [qui] a donné avec une exactitude remarquable et dès les premiers coups, la position de la pièce à très longue portée qui tira sur Paris en mai et juin $1918^{49} \gg$.

Enfin, parmi les scientifiques qui ont déjà quitté Lille bien avant la guerre, 11 est intéressant de mentionner Paul Painlevé et Émile Borel. En 1915, Paul Painlevé est nommé ministre de l'Instruction publique et crée la Direction des inventions intéressant la Défense nationale. À ce titre, il fonde la première flotte aérienne française, donnant une impulsion décisive à l'aéronautique, à l'époque où l'opinion table davantage sur l'avenir des dirigeables que sur celui de l'aviation, perçue comme une curiosité dangereuse. En 1917, il devient ministre de la Guerre, puis président du Conseil, où il réorganise le commandement militaire avec Philippe Pétain et Ferdinand Foch. Il jette les bases d'un commandement interallié et obtient du Président Wilson la promesse de l'envoi d'un million de combattants américains, ceci avant de céder la place à Georges Clemenceau. Émile Borel a, quant à lui, la charge de la Direction des inventions intéressant la Défense nationale. Il nomme, à des fins d'études de balistique, Joseph Kampé de Fériet à la Commission d'artillerie navale de Gâvres dans le Morbihan, où il rencontre Albert Châtelet, Georges Valiron et Arnaud Denjoy. Joseph Kampé de Fériet fondera a Lille l'Institut de mécanique des fluides de Lille après la guerre. La guerre est l'occasion de rencontres et d'échanges entre jeunes scientifiques, qui auront après la guerre la charge de reconstruire les instances scientifiques.

Figure II : « La guerre scientifique », Paul Painlevé, Ministre de l'Instruction publique, dans Excelsior, journal illustré quotidien, le samedi 6 novembre I9Is (collection particulière).

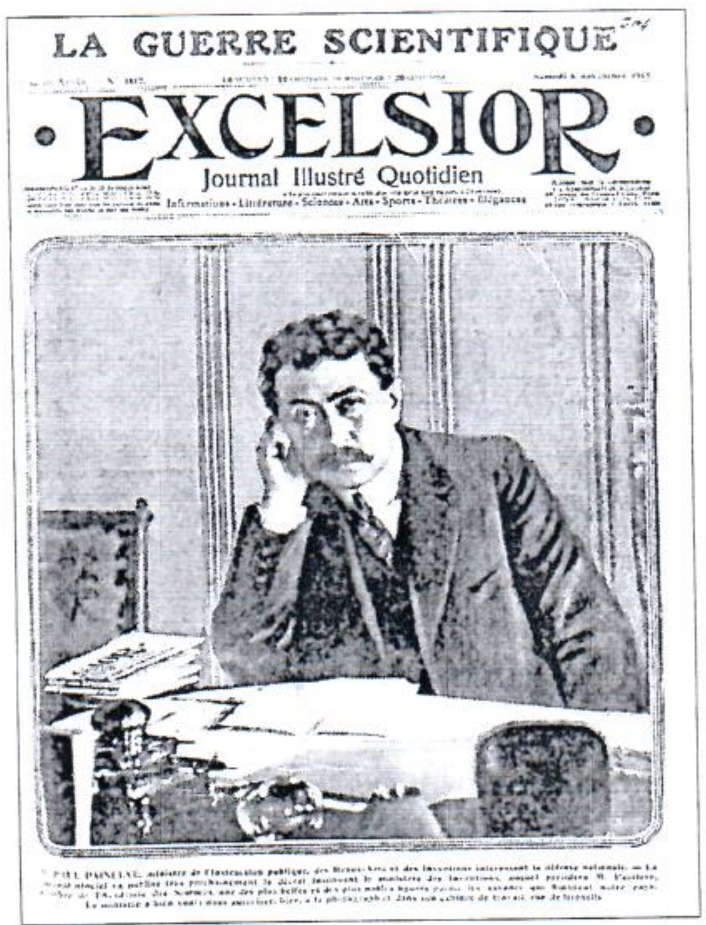

49.- Bulletin des séances de la Société des sciences, de l'agriculture \& des arts de Lille, 1925. 


\section{Une sortie de guerre difficile pour la faculté des sciences de Lille}

La joie de la victoire et de la libération de la France septentrionale est de courte durée car, à mesure que le bilan se précise, la faculté des sciences découvre l'ampleur des destructions.

\section{La désolation humaine}

Après la libération, en octobre 1918, de « quatre ans d'occupation, d'inquiétudes, d'angoisses, de famine $\gg^{50}$, les nouvelles de l'arrière attristent le monde universitaire. Le bilan humain est lourd et les cérémonies mettent en valeur le rôle, le courage et le patriotisme des enseignants et jeunes universitaires lillois pendant la guerre. Selon l'enquête rectorale de 1924 , seize membres du personnel de l'Université de Lille sont morts à la guerre, soit ir \% des mobilisés, dont dix personnels auxiliaires (agents de laboratoire, personnel de secrétariat et de service), et quarante-deux sont blessés, soit $30 \%$ de l'effectif mobilisé. Huit décès et neuf blessés sont regrettés en sciences. Le discours d'allocution du doyen de la faculté des sciences du ir juin 1922 pour l'inauguration de la plaque commémorative des membres de la faculté morts pour la France ${ }^{s 1}$ renseigne sur les disparitions tragiques.

Jean Clairin, professeur de mathématiques et adjudant au $26^{\mathrm{c}}$ régiment d'infanterie territoriale pendant la guerre, est tué par balle dès le 26 août I9I 4 dans un combat de rue à Thun-l'Évêque près de Cambrai. Jean Clairin est issu de la promotion 1896 de l'École normale supérieure (ENS). Il avait trentesept ans. Son triste sort reste longtemps ignoré à Lille puisque son décès n'est annoncé à la Faculté qu'à la rentrée 1916. En 1920, les travaux de Jean Clairin sur « les transformations de quelques équations linéaires aux dérivées partielles du second ordre » et « les invariants des caractéristiques des équations aux dérivées partielles à deux variables indépendantes » sont publiés en tant que Mémoires posthumes dans les Annales de l'ENS, dont il fut l'élève. Paul Lemoult, professeur de chimie, meurt en mai 1916 dans l'explosion de l'usine dont il a la direction. Alphonse Buisine, professeur de chimie, décède en mars 1918 au camp de Milejgany en Lituanie, des suites de sa déportation.

Parmi ces professeurs de la faculté des sciences de Lille disparus de façon violente, Jean Clairin et Paul Lemoult sont des anciens élèves de l'ENS. Les raisons et le contexte de « l'hécatombe normalienne » durant le premier conflit mondial ont été étudiés ${ }^{s^{2}}$. D'autres ne reviendront pas. Henri Deroide disparaît en 1918 d'une «maladie contractée à la guerre ». Bien que non comptabilisés comme « morts pour la France », Charles-Eugène Bertrand,

50.- Auguste PELLET et Albert CHÂTELET, « Éloge funèbre d'Albert Petot, 8 mai 1927 », dans Annuaire des anciens élèves de l'ENS, 1929-1932, p. I4-19.

51.- Bibliothèque municipale de Lille, cote 32616, Université de Lille, Faculté des sciences, Aux professeurs de la Faculté des sciences morts pour la France, 1914-1918. Inauguration de la plaque commémorative, II juin 1922, discours du doyen Chatelet, 16 p.

52.- Nicolas MARIOT, « Pourquoi les normaliens sont-ils morts en masse en 1914-1918 ? Une explication structurale », Pôle Sud, 2012/I, $\mathrm{n}^{\circ} 36$, p. 9-30. 
fules Gosselet ou M. Deligny (chef de travaux de chimie générale), ne sont pas à oublier. Leurs familles sont également touchées. Jules Gosselet et Albert Petot ont chacun perdu leur fils à la guerre. Parmi les chefs de travaux, préparateurs et garçons de laboratoire : Louis Poiron (2I ans), préparateur en chimie générale, meurt lors de la bataille de la Marne en septembre 1914, Raoul Lebrun (2I ans), préparateur en chimie générale, est tué d'une balle près de Le Mesnil-lèsHurlus, Victor Sanson, préparateur en chimie générale, décède dans l'explosion de l'usine de La Pallice en mai 1916. Ernest Verbièse (27 ans), garçon de laborasoire en géologie, est tué lors de la bataille de la Woëvre en avril $1915^{53}$. Élie Ducourant, attaché au laboratoire de chimie, disparaît en juillet I9I9 des suites d'une maladie contractée au service.

Une quarantaine d'étudiants en sciences ont été tués et cent cinquante-quatre sur l'ensemble des quatre facultés, soit près du quart des étudiants mobilisés, ce qui est considérable. En 1919, un père signale au recteur la disparition de son fils, etudiant en sciences, et écrit son chagrin : «Quand je passe rue de Valmy, je ne peux regarder la Maison des étudiants sans avoir les larmes aux yeux. À tous moments, on rencontre des étudiants... Il était si fier de mettre son béret que nous conservons comme une précieuse relique ${ }^{54} \gg$.

\section{La reprise des cours et de la vie universitaire}

La reprise des cours, fixée le 25 puis 31 octobre 1918, se fait dans l'allégresse. Dans la nuit du 16 au 17 octobre 1918, les Allemands quittent Lille. Toutefois, l'enseignement ne reprendra véritablement que le 5 janvier 1919 en raison de la démobilisation tardive des enseignants. Beaucoup d'hommes sont morts 3 la guerre et la relève des enseignants est difficile à assurer. L'Université de Strasbourg, qui était allemande redevient française. Il faut y faire venir des enseignants déjà formés. En 1919, les Lillois Hélö̈se Ollivier, physicien, et Jacques De Lapparent, géologue, partent à l'Université de Strasbourg, ralentissant de fait le redéploiement de l'enseignement supérieur et de la recherche à Lille. En février 1919, dès sa démobilisation, Albert Châtelet est nommé maître de conférences en mathématiques à Lille. Ancien élève de l'École normale supérieure, il est nommé doyen de la faculté des sciences dès 1921 avant d'être nommé recteur de l'académie de Lille en 1924 , succédant ainsi à Georges Lyon ${ }^{55}$. Il faut attendre cette même année pour que l'ensemble des bâtiments et des collections du musée d'Histoire naturelle et du musée houiller soient remis en état, et puissent à nouveau être ouverts au public. Certaines collections sont même enrichies, avec par exemple, les échantillons fossiles prélevés par Paul Bertrand et les séries de paléontologie houillère provenant des bassins lorrains redevenus français. Il faut

53.- Alphonse MALAQUIN, « Rapport sur la situation de l'enseignement supérieur à Lille pendant l'année scolaire 1915-1916 », dans Annales de l'Université de Lille: rapport annuel du Conseil de l'Université..., Lille, Imprimerie Le Bigot, p. 57-58.

54.- AD du Nord, 2 T 653, lettre du 12 décembre 1919, dans Jean-François CONDETTE, «L'Université de Lille dans la Première Guerre mondiale 1914-1918 », Guerres Mondiales et Conflits Contemporains, mars $2000, \mathrm{n}^{\circ} 197, \mathrm{p} .98$.

55.- Voir Jean-François CONDETTE, Albert Châtelet : la République par l'École, Artois Presses Université, 2009, $602 \mathrm{p}$. 
également attendre 1924 pour voir apparaitre de nouveaux instituts : électromécanique en 1924, radiotechnique en 1931, de la houille en 1931, agricole en 1931, de mécanique des fluides en 1930. En 1929, Marie-Louise Delwaulle sera la première femme professeur à Lille, en chimie. Le retour à la normale s'avère donc long et difficile pour la faculté des sciences de Lille très marquée à la fois par la guerre et par une terrible occupation de Lille.

Il y a cent ans, la faculté des sciences était curieusement petite. Ses dimensions et son isolement du reste du pays pendant la guerre permettent d'en avoir un éclairage précis. Les facultés lilloises ont montré un bel exemple de volonté de transmission du savoir et de préservation des jeunes générations, notable dans toutes les civilisations éclairées, quelles que soient les époques. Le plus navrant est que l'Allemagne de 1914-1918 est autant éclairée et cultivée que la France et que le gâchis humain, si ce n'est matériel, ait atteint ce niveau inégalé dans l'Histoire. Les facultés représentent les élites intellectuelles régionales. En concertation avec d'autres instances, elles ont résisté, organisé et pris à bras-le-corps les évolutions nécessaires pour que tout le corps social s'adapte à la pire des situations. Elles donnent ainsi une leçon de vie à tout un chacun et obligent à penser la science et le savoir comme des outils de refus de la folie humaine. 